\title{
Mass spectrometry inside single proteins by atom probe tomography
}

\author{
Shi Qiu ${ }^{1}$, Changxi Zheng ${ }^{2,3}$, Vivek Garg ${ }^{1,4}$, Jian Li $^{5,6}$, Ross K.W. Marceau ${ }^{7 *}$, Jing Fu ${ }^{1 *}$ \\ ${ }^{1}$ Department of Mechanical and Aerospace Engineering, Monash University, Clayton, VIC \\ 3800, Australia \\ ${ }^{2}$ ARC Centre of Excellence for Future Low-Energy Electronics Technologies, Monash \\ University, Clayton, VIC 3800, Australia \\ ${ }^{3}$ School of Physics and Astronomy, Monash University, Clayton, VIC 3800, Australia \\ ${ }^{4}$ IITB-Monash Research Academy, Indian Institute of Technology Bombay, Powai, Mumbai, \\ 400076, India \\ ${ }^{5}$ Biomedicine Discovery Institute, Monash University, Clayton, VIC 3800, Australia \\ ${ }^{6}$ Department of Microbiology, Monash University, Clayton, VIC 3800, Australia \\ ${ }^{7}$ Deakin University, Institute for Frontier Materials, Geelong, VIC 3216, Australia \\ *Corresponding authors. Email: ross.marceau@deakin.edu.au or jing.fu@,monash.edu
}

Atom probe tomography (APT) is considered as the only technique today to provide both the chemical composition and three-dimensional (3D) imaging at a near-atomic resolution [1]. We propose a new APT method to investigate the structure and chemical composition of single proteins maintained in their hydrated state. The specimens are prepared by encapsulating the solution containing proteins with graphene membranes on metal (W) tips (Fig. 1a), with the final tip diameter controlled at less than $100 \mathrm{~nm}$ (Fig. 1b). The hydrated specimens are then transferred to a laser-pulsed atom probe instrument for probing (Fig. 2a).

Ferritin from equine spleen was first imaged by TEM using negative staining protocol, to confirm the consistency of geometry. APT experiments were performed on a LEAP 5000XR instrument (Cameca Instruments, USA) in pulsed-laser mode under ultra-high vacuum $(<1 \times 10$ 11 Torr) with set-point temperature of $35 \mathrm{~K}$. Results have shown that $\mathrm{H}_{2} \mathrm{O}^{+}(18 \mathrm{Da})$ peak can be clearly identified in the acquired mass-to-charge-state ratio spectrum, suggesting that the protein specimen was maintained in the frozen hydrated state (Fig. 2b). 3D maps of the spatial distributions of atomic and molecular species were also reconstructed (Fig. 2b), with the size of the ferritin core and shell consistent with that previously reported [2]. Compared to cryoAPT transfer as recently demonstrated [3], the proposed method demonstrates a new route to investigate the atomic and molecular structures of macromolecules in their near-native state at near-atomic resolution. 


\section{References}

[1] T. F. Kelly, M. K. Miller, Atom probe tomography. Review of Scientific Instruments 78, 031101 (2007).

[2] T. Granier, B. Gallois, A. Dautant, B. Langlois d'Estaintot, G. Precigoux, Comparison of the structures of the cubic and tetragonal forms of horse - spleen apoferritin. Acta Crystallographica Section D 53, 580-587 (1997).

[3] D. K. Schreiber, D. E. Perea, J. V. Ryan, J. E. Evans, J. D. Vienna, A method for sitespecific and cryogenic specimen fabrication of liquid/solid interfaces for atom probe tomography. Ultramicroscopy 194, 89-99 (2018).

[4] The authors acknowledge funding from the Australian Research Council (DP180103955) and also the contributions from Drs Yu Chen, Gediminas Gervinskas and Michelle Dunstone. This work was performed in part at the Melbourne Centre for Nanofabrication (MCN), Victorian Node of the Australian National Fabrication Facility (ANFF). Also, the authors acknowledge the use of facilities within the Monash Centre for Electron Microscopy (MCEM), Monash Ramaciotti Cryo-EM platform, Monash Campus Cluster (MCC), and Deakin University's Advanced Characterisation Facility.
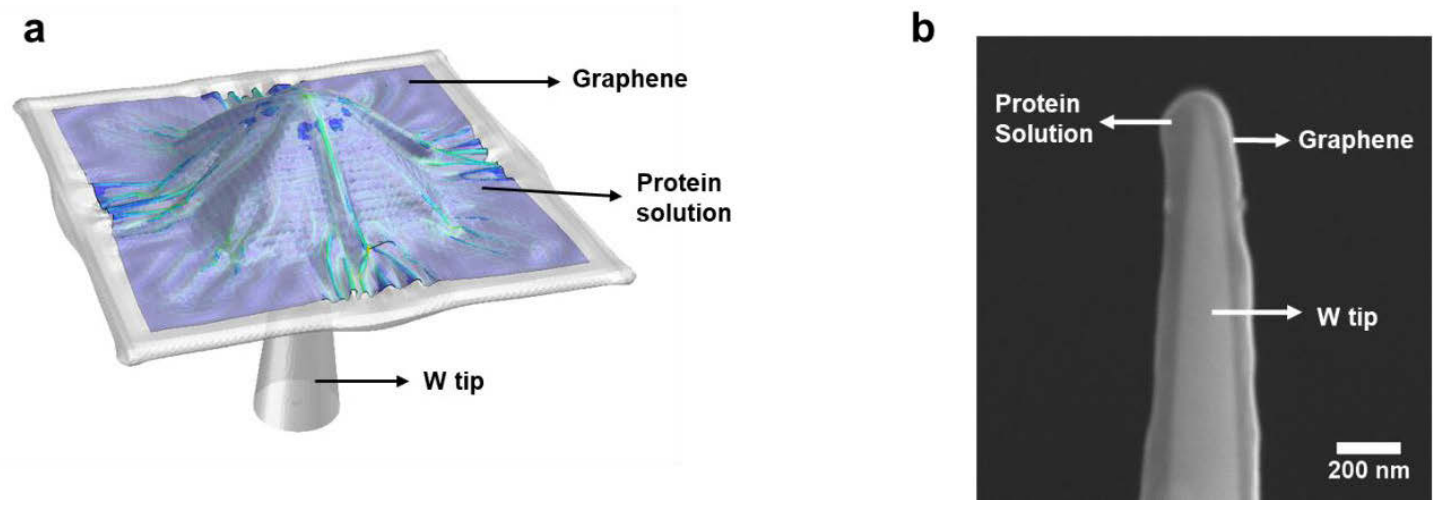

Figure 1. (a) Computer simulation of the dynamic process of encapsulation of protein solution with graphene membrane on an APT specimen tip using finite element method. (b) SEM image of the graphene-encapsulated protein specimen.

a

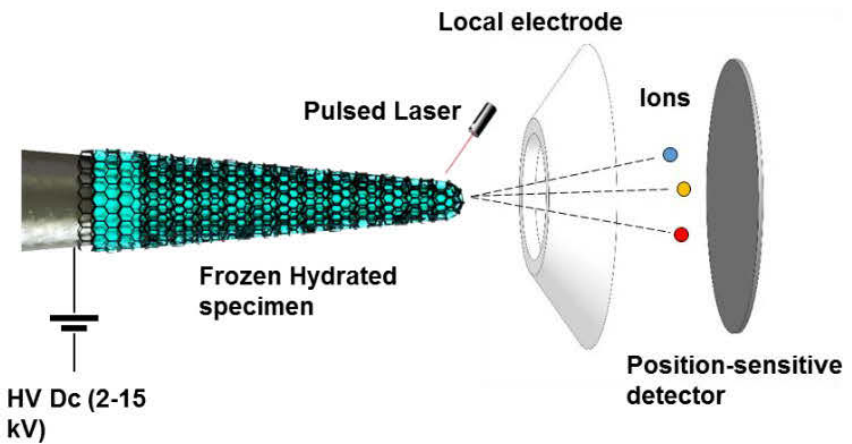

b

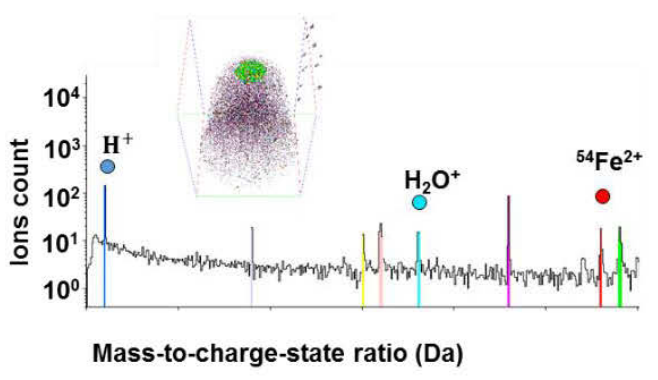

Figure 2. (a) Schematics of the protein specimen probed by laser-pulsed APT in the frozen hydrated state. (b) An acquired mass-to-charge-state ratio spectrum and the associated 3D reconstruction of the field evaporated volume of ferritin at near-atomic resolution. 